\title{
Análisis histórico - epistemológico de nomenclatura Química Inorgánica
}

\author{
Analysis historical - epistemological nomenclature Inorganic Chemistry \\ Paola Díaz Suárez, Dayanni Vargas Rojas y Royman Pérez Miranda
}

Universidad Pedagógica Nacional. pao diazsuarez@hotmail.com; dayannivargas@gmail.com; royman@pedagogica.edu.com

\begin{abstract}
Resumen
Cuando la nomendatura química inorgánica (NQI) se hace objeto de trabajo en el aula persiste la tendencia de abordarla de manera lineal, repetitiva y memorística (Borsese, 1994), por esta razón esta investigación hace énfasis en el análisis histórico - epistemológico de la denominación de sustancias químicas inorgánicas, en el origen de los diferentes sistemas conocidos y aceptados así como la normatividad que actualmente es regulada por instituciones como la IUPAC, vinculando aspectos epistemológicos, históricos y didácticos en su desarrollo, con el fin de aproximar a profesores y estudiantes en formación inicial en química a los estudios históricos que orientan esta temática de modo que se atribuye sentido e importancia al lenguaje de la química.
\end{abstract}

\section{Palabras Clave}

Historia, Epistemología, Nomendatura Inorgánica, Química.

\section{Abstract}

When the inorganic chemical nomendature (NQI) is the subject of dassroom work to address the tendency of a linear, repetitive and memory (Borsese, 1994), for this reason this research focuses on historical analysis - the name of epistemological inorganic chemicals in the origin of the different systems as well known and accepted standards that currently are regulated by institutions such as the IUPAC linking epistemological aspects, historical teaching and its development in order to bring teachers and students in training in chemistry to the historical studies that guide the subject in a way that gives meaning and significance to the language of chemistry.

\section{Keywords}

History, Epistemology, Nomendature Inorganic, Chemistry

\section{Introducción}

El lenguaje es un sistema de recursos para construir significados que proporcionan una semántica como forma particular de crear similitudes y diferencias entre significados. Es necesario porque un concepto empieza a tener sentido cuando se puede relacionar con otros conceptos o ideas, es por esto que el lenguaje se constituye en factor fundamental para la comunicación entre individuos, en particular haciendo más expedita la comunicación entre personas pertenecientes a una misma comunidad cient́fica (Lemke, 1997).

Teniendo en cuenta la importancia de la designación de sustancias inorgánicas en el lenguaje de la ciencia, en especial el de la química y partiendo de la tendencia que se tiene por abordar ésta temática de manera lineal, repetitiva y memoństica, en esta ponencia se hace un análisis cútico del desarrollo histónico - epistemológico de nomenclatura inorgánica bajo una visión Lakatosiana al 
asumir la (NQI) como un programa de investigación cient́fica (PIC) y vinculando la historia social de la ciencia al relacionar aspectos culturales, políticos y económicos en el desarrollo de la misma con el fin de permitir a los docentes y estudiantes en formación inicial crear nuevas metodologías dentro del sistema aula para que la enseñanza del tema objeto de estudio de esta investigación no sea de carácter repetitivo y memorístico sino que se tome de carácter interpretativo.

\section{Desarrollo}

El referente histórico que se aborda para la ponencia tiene como propósito, analizar los documentos de validez histórica a la luz del enfoque lakatosiano con sus programas de investigación cient́fica, estos consisten en reglas metodológicas que pueden ser de heurística negativa o rutas de investigación que sugieren evitarse y de heurística positiva que son los caminos que podrían seguirse (Lakatos, 1978), los programas de investigación permiten la creación y modíficación de la ciencia generando un progreso en el conocimiento aceptando el núdeo firme de heurística negativa de tal forma que este no puede ser modificado y para esto debe estar protegido por un cinturón protector de hipótesis auxiliares el cual recibe los impactos de las contrastaciones y puede ser modificado e induso sustituido completamente (Lakatos, 1978). Por el contrario de asumirse una posición bajo una corriente filosófica inductivista se afirmaría la existencia de enunciados universales y la generación del conocimiento solo por hechos observables, produciendo una linealidad en el conocimiento, ideas que están muy lejanas de lo que los autores conciben como ciencia.

Análisis histórico - epistemológico de nomenclatura química inorgánica

El origen de la nomendatura química inorgánica se remonta desde la Edad Antigua con los aportes realizados por diferentes civilizaciones que se constituían en Egipto, Mesopotamia, China, India y Grecia, pasando por la Edad Medieval con el inicio de la alquimia y la Edad Modema donde se presentan los mayores aportes generados por diferentes científicos dentro de los que se destacan Lavoisier, Dalton y Berzelius, para la consolidación de la nomendatura química inorgánica y finalmente la Edad Contemporánea donde surge la IUPAC que se encarga de unificar por medio de reglas los nombres de las sustancias inorgánicas.

En Egipto se conoció el oro y la plata debido a su alto grado de utilidad para embellecer los templos, palacios y tumbas de las dases más altas que constituían el poder, así como el uso de adomos y bordados que se hacían a los sacerdotes para la decoración de su cuerpo y sus vestidos como los creados al sacerdote levita Aarón hermano de Moisés perteneciente al dero que era la dase social más alta de la época. Estos metales se obtenían por el proceso de levigación que consistía en pulverizar la roca para un posterior lavado en artesas de madera, finalmente se trataba con esponjas las cuales se quedaban adheridas a este recipiente formándose una lámina del metal oro que causaba gran admiración debido al color atractivo que poseía.

Para el año 2.500 a.C. el oro fue llamado $<<N U B>>$ nombre que adquirió porque era explotado de la Nubia (Díaz, 1981), región que queda al sur de Egipto conquistada por el tercer faraón de la dinastía I, según su origen se conocían dos dases de oro, el que provenía de las montañas llamado $<<N U B$ - EN - SET > y el que provenía de los ríos llamado <<NUB - EN - MU>>, por el contrario la plata era llamada <<ELEKTRON>>, además existía otro metal muy conocido el cual se observaba cuando se utilizaba el oro que contenía proporciones altas de plata, por lo tanto se obtenía un metal de color blanco llamado <<ASEM > > $0<<E L E C T R U M>>$ nombre otorgado por otras civilizaciones de Grecia, en donde además los metales como el cobre, estaño, hierro, plata y oro eran usados en la fabricación de vasos, trípodes y vasijas empleados para satisfacer las necesidades de las dases sociales más bajas y los lujos de la nobleza y el dero. 
De la misma manera en el año $\mathbf{2 . 5 0 0}$ a.C. las dinastías egipcias III y VI conocieron otros metales como el cobre al aual se le denomino <<CHOMT>> nombre que correspondería a las aleaciones de este metal, sin embargo en las épocas primitivas lo llamaron $<<$ KALKOS $>>$ y $<<A E S$ CHYPRIUM $>>$ nombre que se le otorgó por encontrarse en la isla Chipre ó <<OPERET >> debido al color que presentaba, pues esta palabra deriva de la palabra aphar, que significa tierra rosada.

Por otro lado Díaz, (1981) sostiene que la civilización egipcia le otorgó nombres como $<<$ CHOMT $>>$ y después se le conoció como $<<M E N>>$, $<<$ TEHSEL $>>$, $<<$ OPHERET $>$, $<<$ OPERET > > y < <BAEN - PET>> que significa hierro del cielo debido a su origen meteórico obtenido a partir de la fundición de minerales como limonita y magnetita.

Figura 1. Algunas representaciones del Código Marcianus López, C. (1984)

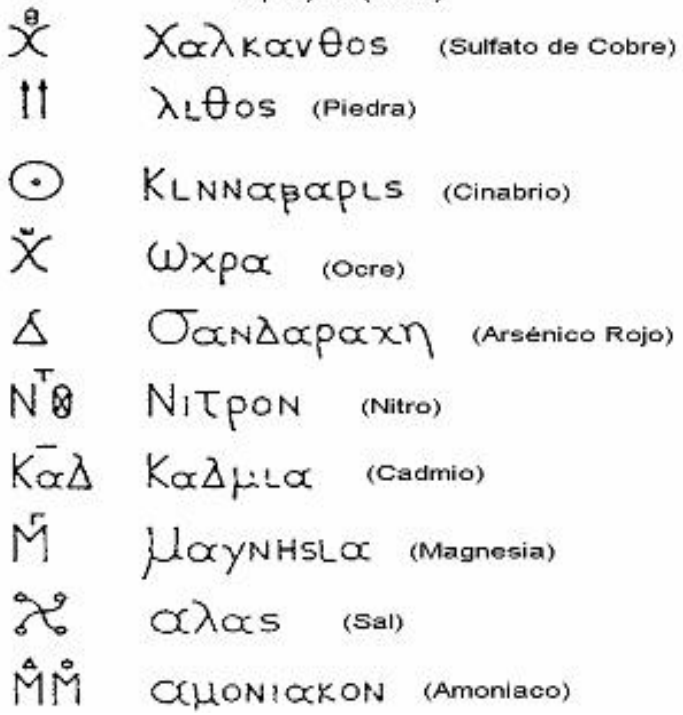

En China se generaron aportes con respecto a la formación de la materia que la concebían como el universo, de allí surge el YIN y el YANG, el primero correspondía al principio femenino, lo negativo, lo pesado y lo seco regido bajo la luna, y el segundo era lo masculino, lo positivo, lo activo, lo ardiente regido bajo el poder del sol, teoría que generó la idea que decía que la materia estaba constituida por cinco elementos tales como: el metal, la madera, la tiena, el agua y el fuego (Brock, 1992).

Más adelante en la civilización griega se dan dos escuelas de pensamiento, la materialista y la espiritualista, la primera y más importante surge en Jonia que representa el primer intento conocido de brindar una descripción del universo sin recurrir a fuerzas superiores en donde surge una lista de símbolos para sustancias químicas y palabras téonicas (López, 1984), de ahí la existencia del

Código Marcianus (Figura 1).

De otro modo según Díaz, (1981), la cultura babilónica perteneciente a Mesopotamia, pudo observar el cosmos relacionándolo con la humanidad a partir del horóscopo, de esto se decía que todo en la tiena estaba regido por los cinco planetas (Figura 2), el sol y la luna induido los metales que eran importantes en el común de la humanidad y se relacionaban a demás con los días de la semana, sin embargo al adicionar a las diferentes formas un rasgo en particular se podían presentar diferentes sustancias, así por ejemplo al adicionarle tres líneas cortas a la parte cónica del símbolo oro se representaba la limadura de oro, diferente de cómo se presentaba la limadura de plata debido a que las líneas largas cubrían la luna que representaba la plata. Un rectángulo en la parte inferior de los símbolos de estos metales representaba las

Figura2. Simbolos alquimistas para los metales
López, C. (1984)
\begin{tabular}{|l|l|l|}
\hline METAL & PLANETA & SIMBOLO \\
\hline Oro & Sol & \\
\hline Plata & Luna & \\
\hline Hierro & Marte & \\
\hline Cobre & Venus & \\
\hline Plomo & Saturno \\
\hline Estaño & Jupiter & \\
\hline Mercurio & Mercurio \\
\hline
\end{tabular}
hojas de los mismos.

Para los alquimistas, su objetivo principal era el de trasmutar los cuatro metales viles o bajos: cobre, hierro, plomo y estaño en metales nobles: oro y plata, para esto se utilizaba el plomo fundido y ennegrecido, sin embargo este fue sustituido por el mercurio fluido a temperatura 
ambiente. Para poder trasmutar estos elementos era necesaria la presencia de la "piedra filosofal" o "elixir de la vida" el cual debía eliminar las enfermedades, devolver la juventud, prolongar la vida e induso asegurar la inmortalidad; y en presencia necesariamente de un disolvente simbolizado en varias ocasiones con un dragón (Díaz, 1981).

La particularidad de estos alquimistas al escribir en un lenguaje daro era de gran relevancia en la época, sin embargo la tendencia a ocultar la ciencia en la que ellos participaban conllevo a que sus publicaciones y prácticas tuvieran un lenguaje ocultista en cuanto al nombramiento de las sustancias, debido a esto algunas sustancias y elementos cambiaron sus nombres, generando que la sociedad ignorará este lenguaje, desconocimiento que forjaría un atraso cultural. A partir de esto se dice que los alquimistas mostraban una posición positivista al creer que eran los poseedores de la verdad absoluta, por lo tanto decidieron utilizar una nueva simbología, en donde se propagaba la confusión y el misterio haciendo que la alquimia no tuviera un progreso rápido y avanzado, pero constituyendo el primer paso para la consolidación de un lenguaje propio de la química.

\section{Paracelsus y la Iatroquímica}

En el siglo XV el continente Europeo se caracterizaba por el alto índice de mortalidad a causa de las malas condiciones sanitarias de la época, debido a que la medicina estaba poco desarrollada y la higiene era casi nula se desarrollaron enfermedades como la peste, la lepra, el tifus y la sífilis traída por los mercenarios desde Italia. Para esta época Paracelsus (1493 - 1541) fundador de la iatroquímica o rama de la química y la medicina preparó el "ALCAHEST", un remedio casero que según él era capaz de curar todas las enfermedades entre ellas la sífilis que según Paracelsus debía ser curada por los "ALCAHEST" espećficos. Por otro lado Paracelsus logra hacer la diferenciación entre alumbres y $<<$ VITRIOLOS $>>$ que se denominaban igual en aquella época, además fue el primero en introducir el término "alcohol", correspondiente a la sustancia denominada $<<$ ESPIRITU DE VINO>>.

Un seguidor de Paracelsus e iatroquímico fue Van Helmont (1577 - 1614) o también llamado "filosofo del fuego", quien Introdujo la palabra <<GAS>> del latín Chaos, (carente de forma). Al calentar $28 \mathrm{Kg}$. de carbón vegetal y luego al quedar expuesto al aire el carbón quedó reducido a $2.2 \mathrm{Kg}$. de cenizas, el resto había desaparecido en forma de gas "gas carbónico", al que llamo $<<$ ESPIRITU SIIVESTRE >>.

De otro lado uno de los aportes que realizó Glauber (1604 - 1670), a la nomendatura química fue la distinción que hizo entre los ácidos sulfuroso <<SPIRTIUS VOLATILIS VITRIOLI>> y acido sulfúrico <<OLEUM ACIDUM VITRIOLI>>. Preparó el acido nítrico <<SPIRITUS NITRI >> y el <<ACIDO MURIATICO>> (acido clorhídrico) ó <<SPIRITUS SALIS >>.

\section{La caída de la Iatroquímica con el surgimiento del flogisto}

La capacidad que tenia un cuerpo para arder se debía, según esta teońa a la existencia en su composición de una determinada y especifica sustancia llamada flogisto (Gutiérrez, 1994). De acuerdo con la doctrina del químico y medico Alemán Stahl (1660 - 1743), si una sustancia ardía o algún metal se calcinaba se producía flogisto.

Stahl explicó la combustión del azufre y su reauperación después de tratarlo con $<<S A L$ DE TARTARO>> "carbonato potásico":

$$
\begin{gathered}
\text { Azufre } \rightarrow \text { ácido universal }+ \text { flogisto } \\
\text { Acido universal }+<<\text { SAL DE TÁRTARO }>\rightarrow<<\text { TARTARO VITRIOLADO }>> \\
<<\text { TÁRTARO VITRIOLADO }>+ \text { carbón vegetal } \rightarrow \text { azufre }
\end{gathered}
$$

Por su parte Joseph Black (1728 - 1799) reconoció la existencia de un gas en algunos minerales hoy conocidos como carbonatos que se desprendía de ellos por calentamiento y ayyas propiedades 
eran distintas a las del aire común a la que le dio el nombre de <<AIRE FDADO>> por ser fijado por la cal, actualmente este aire es conocido como dióxido de carbono.

Black considera el doro como uno de los cuerpos más notables de la química, en 1774 Mr. Scheele (1742 - 1786) le da el nombre de <<ACIDO MURIATICO DESFLOGISTICADO>>, auando Black le añadió oxígeno al <<ÁCIDO MURIÁTICO>> y al observar cambios de aspecto y propiedades lo llama <<ACIDO OXIMURIATICO>> (Díaz, 1981).

Figura 3. Simbolismo de Geoffroy para ácidos y bases Mosquera, C. (2000)

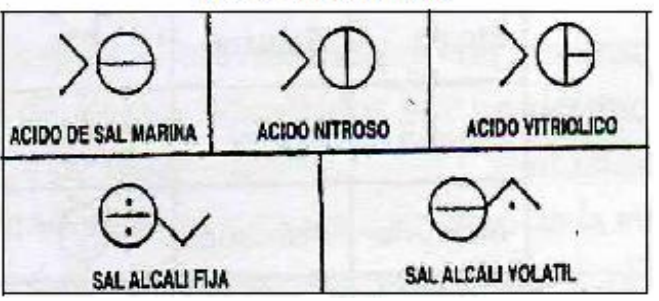

Mas adelante, Geoffroy (1672 - 1731) retoma el simbolismo alquimista (Figura 3 ) representando los ácidos y las bases, aunque con algunas adiciones (Mosquera, 2000).

Los símbolos de Geoffroy sirvieron de inspiración para muchos trabajos como el realizado por Bergman (1734 - 1784), que retoma algunos de sus símbolos y reconoce en 1775 el carácter acido de una disolución de gas carbónico, además tiene del aire una concepción exacta al considerarlo una mezda de tres fluidos, el <<ACIDO AEREO>> (gas carbónico), el <<AIRE VICIADO>> (nitrógeno) y el <<AIRE PURO $>$ (oxígeno). Además a Bergman se le debe el inicio del simbolismo químico especialmente para representar ácidos y álcalis. Tomando viejos símbolos alquímicos, añade una $\oplus$ para significar ácido; el símbolo † para sales de los metales (óxidos); para los álcalis $\Theta$; el <<AIRE FLOGISTICADO >> (oxígeno), se representa por el símbolo (Díaz, 1981). Los trabajos de Bergman fueron de gran importancia para la creación de una nueva terminología química basada en la visión modema de la composición.

En 1774 Priestley comunicó a Lavoisier que había recogido una nueva dase de aire al calentar la $<<$ CAL ROJA DE MERCURIO>>, este aire favorecía más la combustión que el aire común. Comunicó sus resultados hasta un año después, y dio al nuevo aire el nombre de <<AIRE DESFLOGISTCADO >> u oxígeno.

\section{Derrota del flogisto y surgimiento de la revolución química}

En el siglo XVIII Lavoisier (1743 - 1794) ataca los constructos del flogisto sin contemplaciones, tras encontrar varias dificultades en esta teoría como la explicación poco satisfactoria que tenían los seguidores del flogisto al afirmar que cuando un metal se caldinaba perdía su flogisto y la cal que quedaba pesaba mas que el metal original, algo muy contradictorio que un cuerpo gane peso por la perdida de parte de su composición (Gutiérrez, 1994). De manera que una vez exduida la idea del flogisto, Lavoisier produce una verdadera revolución química. Guyton, Berthollet, Fourcroy, G Monge, A. Seguin y $\mathbf{N}$. $L$ Vauquelin se unieron a las ideas de Lavoisier y decidieron vincularse a la revista Annals de Chimien fundada por Lavoisier y su joven disápulo Pierre Adet (1763 - 1834), en el año 1789.

La gran preocupación que tenia Lavoisier y sus seguidores era que hasta ahora el lenguaje de los químicos había llegado a ser bastante confuso y pintoresco, debido a la ausencia de unas normas mínimas adoptadas, así nombres como <<ACEITE DE VITRIOLO $>>$, $<<$ CREMA DE TARTARO $>>$, <<MANTECA DE ANTIMONIO >>, <<AZAFRÁN DE MARTE $>>,<<$ SAL AMARGA $>$, $<<A Z U C A R$ DE SATURNO>>, recordaban mas el lenguaje del arte culinario que el de una ciencia (Gutiérrez, 1994).

En 1782 Guyton de Morveau (1737 - 1816) postuló un sistema de nomendatura en el libro Methode de Nomendature Chimie en colaboración con Lavoisier y los demás autores, éste fue publicado en 1787, gran parte del libro consiste en un diccionario identificando el nuevo nombre de las sustancias y el antiguo, así gracias a las traducciones, se convirtió rápidamente en el lenguaje 
Figura 4. Símbolos ideados por Hassenfrantz y Adet. López, C. (1984)

\begin{tabular}{|c|c|c|c|}
\hline NITKOGCNO & $A C E 1410$ & A & CCRAE \\
\hline POI ASA & OLALATO & 0 & PLCKO \\
\hline $\operatorname{Sos} A$ & CITRATO & $c$ & CINC \\
\hline$C A L$ & LACTATO & 6 & ARIIMONIO \\
\hline
\end{tabular}

intemacional de la química (Brock, 1992). De manera que con la publicación del Methode de Nomendature Chimie se establece un lenguaje propio de la química al desaparecer nombres como el <<ACEITE DE VITRIOLO $>>$ que pasa a ser el ácido sulfúrico, <<EL ESPÍRITU DE VENUS>> (ácido acético); <<EL AZAFRÁN DE MARTE > > u óxido de cinc; <<EL VITRIOLO DE CHIPRE >> ó sulfato cúprico, entre otros, lo que favorece la consolidación de la química como ciencia bajo una visión Lakatosiana.

De otro lado Hassenfrantz (1755 - 1827) y Adet (1763 - 1832) retoman nuevamente la simbología al representar los productos químicos mediante figuras geométricas los elementos eran líneas rectas con distintas indinaciones, los metales representados como cárculos y los álcalis como triángulos (Díaz, 1981).

Del mismo modo Dalton (1766 - 1844) adoptó la idea de representar cada elemento con un símbolo pero no perduraron seguramente porque implicaban un gasto bastante alto en imprenta, la idea del simbolismo de Dalton es perfeccionada por Berzelius (1779 - 1848) quien ideó una nueva dasificación de las sustancias en latín, al respecto describió que los símbolos químicos debían ser letras para facilitar la escritura, así en este mismo año introdujo un simbolismo racional basado en los nombres de los elementos en latín.

Los compuestos se indicaban mediante el signo de la suma entre los constituyentes, como en el oxido de cobre $(\mathrm{Cu}+\mathrm{O})$ ó el agua $(2 \mathrm{H}+\mathrm{O})$, donde el elemento electropositivo era el que aparecía en primer lugar. Mas adelante, Berzelius prescindió del signo más y puso los dos elementos uno junto al otro. Los diferentes números de los elementos estaban, entonces, indicados por superíndices, por ejemplo $\mathrm{S}^{2} \mathrm{O}^{3}$ era la molécula de <<ÁCIDO SULFURICO〉>, este simbolismo algebraico solo empezó a ser usado en la década de 1830, sin embargo, en la actualidad se utilizan los subíndices propuestos por liebig (1803 - 1873) en 1834, solo los químicos franceses siguieron utilizando superíndices hasta el siglo XX.

A los compuestos formados por oxígeno Berzelius los redujo a un punto sobre su congénere electropositivo de este modo $\dot{\mathrm{C}}=\mathrm{Cu}+\circ$ poco después lo cambio por una tilde para indicarlo así Cú. En 1827 Berzelius introdujo subrayados o "barrados para indicar dos átomos de un elemento, por ejemplo los símbolos del agua y el alumbre potásico se expresaban así:

$$
\begin{gathered}
H O \underline{H} \\
\dot{\mathrm{K}} \ddot{\mathrm{S}}+\ddot{A} \mathrm{I} \dot{S}+24 \dot{H}
\end{gathered}
$$

La IUPAC en la organización de la nomenclatura química inorgánica

La necesidad de uniformidad de las prácticas entre los químicos de habla Inglesa fue reconocida en 1886 y dio lugar a acuerdos sobre su uso por las sociedades British American Chemical. En 1913, el Consejo de la Asociación Internacional de Sociedades Químicas nombró una comisión de nomendatura para compuestos inorgánicos y orgánicos, pero la Primera Guerra Mundial terminó abruptamente sus actividades. El trabajo fue reanudado en 1921, cuando la IUPAC (Unión Internacional de Química Pura y Aplicada), en su segunda conferencia nombró comisiones para la nomendatura de los compuestos inorgánicos, orgánicos, químicos y biológicos (Connelly, 2005), tales como la Comisión de la nomendatura de química orgánica (CNOC) y la Comisión de la 
nomendatura de Química Inorgánica (CNIC) y generó publicaciones que se dan en cierto tiempo donde se refleja información de las nuevas correcciones. Estos aspectos tienen ventajas en este programa de investigación al crearse una comunidad espećfica con funciones definidas que contribuyen dentro de su evolución.

El primer informe de la comisión de nomendatura para compuestos inorgánicos en 1940, generó conciencia sobre la necesidad de un desarrollo más sistemático en la nomendatura. Algunas de las características mas importantes de ese informe fue la aprobación del sistema stock para indicar los estados de oxidación, el establecimiento para citar los compuestos binarios en las formulas y en los nombres, y el desarrollo de prácticas uniformes para nombrar diferentes compuestos. Estas revisiones de la IUPAC fueron examinadas y publicadas en un pequeño libro en 1969, seguida de una revisión en 1971 y un suplemento titulado como nombrar las sustancias inorgánicas en 1977. En 1990 las recomendaciones de la IUPAC, fueron revisadas nuevamente con el fin de reunir los cambios que se habían realizado en los últimos 20 años (Connelly, N. 2005).

En la actualidad se han introducido numerosas modificaciones en las normalizaciones terminológicas dado que se han conocido aproximadamente 100.000 a 150.000 millones de compuestos químicos. La función de realizar estas modificaciones están en manos de los especialistas en nomenclatura que tienen como función codificar las normas establecidas a fin de que toda persona puede utilizarlas para identificar las sustancias, dentro de estas normas se estableció más de una forma de nombrar las sustancias, pero siempre destacándose el nombre mas común como por ejemplo para el compuesto con formula molecular $\mathrm{HCl}$ es mas conocido el nombre de ácido dorhídrico que el de cloruro de hidrogeno.

\section{Conclusiones}

El análisis histórico - epistemológico de la nomenclatura química inorgánica muestra posiciones epistemológicas desde el empirismo con las antiguas civilizaciones pasando por los alquimistas con una visión positivista, seguida de la revolución química de Lavoisier que se diferencia de las anteriores con una visión deductivista de la ciencia con la creación de un programa de investigación de heurística positiva al igual que la IUPAC.

La creación de un nuevo sistema de nomendatura química inorgánica tuvo impactos a nivel cultural, político y económico, aspectos vinculados a la historia social principalmente del continente europeo, porque a partir de éstos se constituían las nuevas formas de pensamiento.

La institucionalización de diversos sistemas de nomendatura a través del tiempo genera desventajas en el programa de investigación debido a que no establece un sistema universal para la designación de las sustancias inorgánicas creando confusiones y divergencias en el lenguaje de la comunidad especializada, sin embargo, se destaca del nuevo sistema la consolidación de un lenguaje sin ambigüedades que permite nominar bajo los mismos criterios las diferentes sustancias.

Finalmente, el análisis histórico - epistemológico de la nomendatura química inorgánica permite a los docentes y estudiantes en formación inicial reflexionar acerca de la manera como se vincula la historia y la epistemología de la ciencia dentro del sistema aula al abarcar una temática, lo que posibilita una reducción en la memorización por parte de los estudiantes.

\section{Bibliografía}

Borsese, A (1994). Il problema de lla Comunicazione Linguistica a Savola: II Linguaggio Scientifico e Chimico in Particolare. Enseñanza de las ciencias, 12 (3), 333 - 337. 
Brock, W. (1992). Historia de la Química. Madrid, España: Alianza Editorial.

Díaz, H. (1981). Contribución al Estudio del Desarrollo Histórico de la Nomenclatura Química. Tesis de Pregrado. Universidad Nacional de Colombia. Bogotá - Colombia.

Gutiérrez, P. (1994). Método de la nueva nomendatura química de M. M. Morveau, Lavoisier, Bertholet y de Fourcroy. Madrid, España: Editorial Fundación de Ciencias de la Salud.

Lakatos, I. (1978). La Metodología de los Programas de Investigación Madrid, España: Alianza editorial S.A.

Lemke, J. L. (1997). Aprender a Hablar Ciencia: Lenguaje, Aprendizaje y Valores. Barcelona: Editorial Paidos.

Connelly, N. (2005). Nomendature of Inorganic Chemistry IUPAC Recommendations 2005. Editorial RSC Publishing.

Mosquera C. (2000). Análisis Histórico - Epistemológico de las Representaciones Simbólicas y la Terminología química: Implicaciones Didácticas de orientación Constructivista. Bogotá: Universidad Distrital Francisco José de Caldas. 